\title{
A NOTE ON THE LOCALIZATION THEOREM FOR PROJECTIVE MODULES
}

\author{
CLAYTON SHERMAN
}

\begin{abstract}
Let $R$ be a ring and $S$ a central multiplicative subset. An example is given to show that the localization theorem for projective modules, valid when $S$ consists of non-zero-divisors, does not hold when $S$ is allowed to contain zero-divisors.
\end{abstract}

Let $R$ be a ring with 1 , and $S$ a multiplicatively closed central subset. Let $H$ denote the (exact) category consisting of those finitely generated $R$-modules $M$ admitting a resolution of length one by finitely generated projective $R$-modules, and such that $M_{S}=0$. When $S$ consists of non-zero-divisors, Quillen and others have established a long exact localization sequence $([1]-[3])$

$$
\ldots \rightarrow K_{1} H \rightarrow K_{1} R \rightarrow K_{1} R_{S} \rightarrow K_{0} H \rightarrow K_{0} R \rightarrow K_{0} R_{s}
$$

The purpose of this note is to give a class if simple examples showing that, in general, the hypothesis on $S$ cannot be relaxed.

Let $R$ be any commutative Noetherian local ring with $\operatorname{dim} R>1$, depth $R$ $=0$. Let $f$ be any nonnilpotent element of $R$, and put $S=\left\{1, f, f^{2}, \ldots\right\}$. By hypothesis, all elements of the maximal ideal of $R$ are zero-divisors. Then a simple argument ([4, Lemma 4, p. 182]) shows that any finitely generated $R$-module of finite projective dimension is, in fact, projective (and consequently, free). Thus $H=0$ in this case. If an exact sequence existed in the form above, it would follow that the $\operatorname{map} K_{n} R \rightarrow K_{n} R_{S}$ would be an isomorphism for $n \geqslant 1$.

Now, since $R$ is local, $K_{1}(R)=R^{*}$, where $R^{*}$ is the multiplicative group of units of $R$. Consider $1 / f \in R_{S}^{*} \subset K_{1}\left(R_{S}\right)$; we claim that $1 / f$ is not in the image of $R^{*} \rightarrow R_{S}^{*} \subset K_{1}\left(R_{S}\right)$. For otherwise there would exist $a \in R^{*}$ such that $a / 1=1 / f$ in $R_{S}$. This implies that $f^{n}(1-a f)=0$ in $R$ for some $n>0$. But 1 - af is a unit, so $f^{n}=0$, a contradiction.

As an example, let $k$ be a field, and put $R=A_{m}$, where $A=$ $k[x, y] /\left(x^{2}, x y\right)$, and $m$ is the maximal ideal generated by the images of $x$ and $y$ in $A$; let $f$ be the image of $y$ in $R$.

Received by the editors October 28, 1978.

AMS (MOS) subject classifications (1970). Primary 18F25.

() 1979 American Mathematical Society 0002-9939/79/0000-0303/\$01.50 


\section{REFERENCES}

1. S. Gersten, The localization theorem for projective modules, Comm. Algebra 2 (1974), 307-350.

2. D. Grayson, $K$-theory of hereditary categories, J. Pure Appl. Algebra 11 (1978), 67-74.

3. __ Higher algebraic K-theory: II (after Quillen), Algebraic $K$-Theory (Evanston, 1976), Lecture Notes in Math., vol. 551, Springer-Verlag, New York, 1976.

4. I. Kaplansky, Fields and rings, 2nd ed., Univ. of Chicago Press, Chicago, 1972.

Department of Mathematics, New Mexico State University, Las Cruces, New Mexico 88003 